\title{
Nonlinear control of light in periodic photonic structures: from waveguides to cavities
}

\author{
(Invited Paper) \\ Dragomir N. Neshev, ${ }^{1}$ Nicolas Marsal, ${ }^{2}$ Delphine Wolfersberger, ${ }^{2}$ Marc Sciamanna, ${ }^{2}$ Germano Montemezzani, ${ }^{2}$ \\ Andrey A. Sukhorukov, ${ }^{1}$ Wieslaw Krolikowski, ${ }^{1}$ and Yuri S. Kivshar ${ }^{1}$ \\ ${ }^{1}$ Nonlinear Physics Centre and Laser Physics Centre, Research School of Physical Sciences and Engineering, \\ Australian National University, Canberra, Australia \\ ${ }^{2}$ Laboratoire Matériaux Optiques, Photoniques et Systmes (LMOPS), CNRS UMR 7132, \\ Unité de recherche commune à Supelec et Université Paul Verlaine de Metz, France.
}

\begin{abstract}
We review the fundamentals of light control in nonlinear periodic photonic lattices. In particular, we demonstrate their ability to control the modulational instability and pattern formation in a nonlinear dissipative feedback system.
\end{abstract}

\section{Photonic Lattices}

Periodic photonic structures exhibit spatial variations of their refractive index on a wavelength scale. Due to the light scattering from this periodic refractive index modulation, the dispersion properties of optical fields are strongly modified. This enables the engineering of the structural properties, facilitating observation of novel effects on manipulation of light refraction and diffraction. An important consequence of the strong Bragg scattering inside the structure is the appearance of photonic bandgaps: forbidden zones of frequencies or propagation constants at which light propagation is inhibited. The presence of photonic bandgaps influences significantly the interaction of light with the material at high intensity. New types of nonlinear localisation appear when the nonlinear change of the refractive index shifts the propagation constant inside the photonic bandgap, allowing for novel type of nonlinear switching and formation of gap solitons [1].

Various different platforms for study of nonlinear effects in periodic structures have been utilized, however most of them require the use of high-power lasers and are not suitable for fast prototyping. Recently, the platform of optically induced lattices in photorefractive (PR) crystals [2] has gain large attention due to the possibility for dynamic tuning of lattice periodicity, depth, and geometry, as well as low power requirements. In this platform, several broad beams are sent into a PR crystal, forming an interference pattern [Fig. 1(a)] that induces a periodic refractive index modulation in transverse $x$ direction. The light with nonzero transverse wavevectors will then scatter from this induced lattice leading to formation of a bandgap structure, see Fig. 1(b). The use of this platform has pioneered the demonstration of a number of fundamental nonlinear effects, including excitation of gap solitons, and nonlinear surface waves [3].

Despite the intensive experimental research that followed from the concept of photonic lattices, all existing experiments are performed in conservative systems (waveguide array geometry) without a feedback or gain. Therefore, a natural question

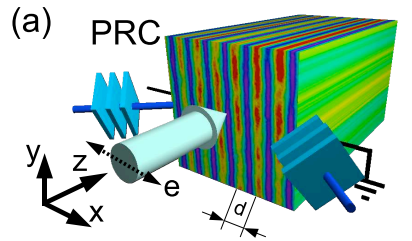

(b)

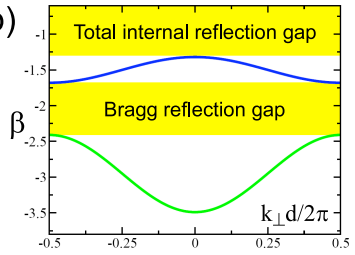

Fig. 1. (a) Optical induction of a 1D photonic lattice in a photorefractive crystal (PRC). (b) Typical Bloch-wave dispersion of 1D lattices.

arises: Can the concept of photonic lattices be generalised for studies of dissipative nonlinear periodic systems with a feedback? Here we demonstrate experimentally this generic concept and apply it for control of the modulational instability of cavity modes and patterns.

\section{Photonic LATtice in A CAVITy}

In a dissipative system, the unique interplay between nonlinearity, dispersion, gain, loss, and feedback results in a large number of unstable pattern modes. Due to the feedback however, only few of these modes normally grow, leading to cavity modulational instability (MI). The control of the cavity MI forms an important problem in optics and various different techniques based on spatial filtering have been suggested.

The implementation of photonic lattices inside the cavity has also been explored theoretically and has been shown to lead to novel nonlinear phenomena, including discrete cavity MI [4] and discrete cavity solitons [5]. Despite the large theoretical interest however, an experimental demonstration of the interplay between periodic photonic lattices and optical patterns in a feedback system is still lacking.

Here, we present an experimental study of the control of MI in a feedback nonlinear system by a one-dimensional (1D) photonic lattice. We combine two concepts: a photorefractive two-wave mixing in a single-mirror configuration [6] and an optically induced lattice [2], and study how the strength and periodicity of the lattice influence the conditions for MI, and correspondingly the pattern formation.

\section{A. Experimental setup}

Our experimental setup consists of two intrinsic parts. The part indicated by the solid line in Fig. 2, called a "pattern 


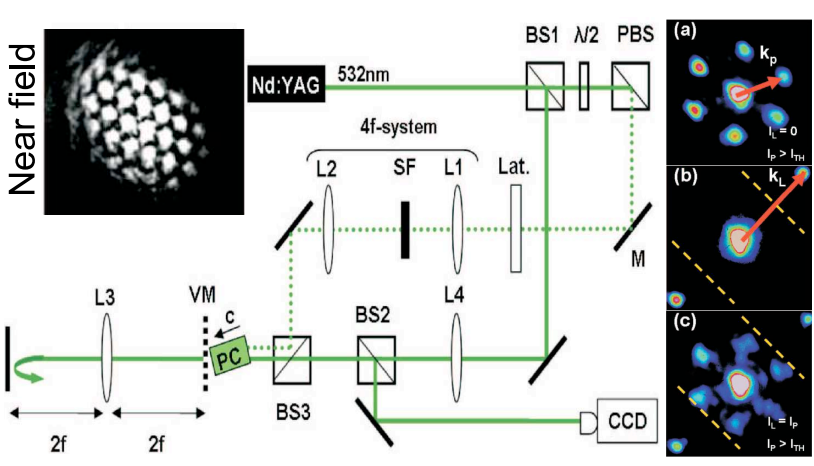

Fig. 2. Experimental setup. L - lenses, PBS - polarising beam splitter, SF spatial filter, Lat - 1D grating, $\lambda / 2$ - half-wave plate, $M$ - mirror, VM - virtual mirror, PC - $\mathrm{BaTiO}_{3}$ PR crystal. Inset: typical near-field pattern. Right: Farfield patterns: (a) hexagonal pattern without lattice, (b) linear diffraction on the lattice, (c) co-existence between nonlinear pattern and linear diffraction for $k_{L} \simeq 2.2 k_{P}$. Dashed lines denote the position of the lattice bandgap.

beam", represents a standard PR two-wave mixing experiment [6] with a tunable single feedback. The crystalline c-axis of the undoped $\mathrm{BaTiO}_{3}$ crystal points towards the feedback mirror, but is rotated by roughly $25^{\circ}$ with respect to the pattern beam. At a fixed mirror-crystal distance, a hexagonal pattern is formed above a threshold intensity $I_{\mathrm{TH}}$ of the pattern beam. A typical near-field intensity distribution $I_{\mathrm{P}}$ is shown in the inset of Fig. 2(top). The lattice forming beam is polarized orthogonally to the pattern beam and is shown with a dotted line in Fig. 2. It is created by a diffraction of a Gaussian beam onto a $1 \mathrm{D}$ grating. The $\pm 1^{\text {st }}$ diffraction orders are selected and recombined inside the $\mathrm{PR}$ crystal by a $4 f$ system. The patterns are being identified by monitoring their far-field distribution.

Figure 2(a) shows a typical far-field hexagonal pattern formed in the absence of the lattice above a threshold power for pattern formation $(20 \mathrm{~mW})$. The lattice strength, on the other hand, is tested by temporarily removing the feedback mirror and monitoring the pattern beam diffraction on the lattice in the far-field. As seen in Fig. 2(b), this diffraction gives rise to the two outer spots along the diagonal (obtained at $45^{\circ}$ lattice orientation), while the central spot corresponds to the zero-order diffraction. The arrows in Fig. 2(a,b) represent the transverse wavevectors of the hexagonal pattern $\left(k_{\mathrm{P}}\right)$ and the lattice $\left(k_{\mathrm{L}}\right)$. The dashed lines in Fig. 2 correspond to the edges of the first Brillouin zone of the lattice, situated at $k_{\mathrm{L}} / 2$.

Next, we investigate the effect of the relative magnitude and orientation of $k_{\mathrm{L}}$ on the formation of patterns in the system. In the experiments, we create the periodic lattice and then we launch the pattern beam into the medium.

\section{B. Control of MI in dissipative feedback systems}

First we set the lattice periodicity such that $k_{\mathrm{L}} \simeq 2.2 k_{\mathrm{P}}$, hence all the wavevectors of the instability modes fall within the first Brillouin zone of the lattice [Fig. 2(c)]. In this case, the nonlinear hexagonal pattern co-exists with the 1D lattice diffraction. Note that in this case the optical power of the pattern beam $(\simeq 30 \mathrm{~mW})$ is larger, because the presence of the lattice in the PR crystal tends to increase the hexagonal pattern threshold.

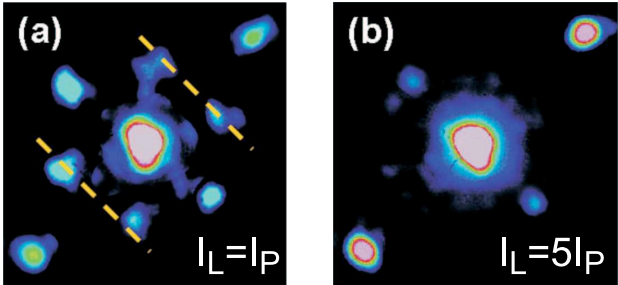

Fig. 3. (a,b) Bandgap inhibition of instability modes for for $k_{\mathrm{L}} \simeq \sqrt{3} k_{\mathrm{P}}$ and two different lattice intensities. $I_{\mathrm{P}}>I_{\mathrm{TH}}$.

An important condition for bandgap control of the patterns is realised when the periodicity of the lattice is such that $k_{\mathrm{L}}=\sqrt{3} k_{\mathrm{P}}$. In this case, the propagation constant hexagonal instability modes are situated exactly inside the bandgap region of the lattice, as seen in Fig. 3(a) for comparable pattern and lattice beams intensities. By increasing the lattice beam intensity, $I_{\mathrm{L}}=5 I_{\mathrm{P}}$ [seen by the two brighter outer spots in Fig. 3(b)], the MI can be suppressed in the bandgap region [Fig. 3(b)] due to the fact that the lattice bandgap prohibits the growth (from noise) of instability modes with corresponding propagation constants. Qualitatively similar effect occurs if two spots of the hexagons overlap the bandgap area for $k_{\mathrm{L}} \simeq 2 k_{\mathrm{P}}$, again leading to symmetry breaking of the induced patterns. It is important to note that the output differs drastically, when the lattice beam is sent through the crystal after the formation of the pattern. In this case, the established high intensity instability modes shift the lattice bandgap such that the propagation constant of the modes lies outside the bandgap region and suppression of the instability is no longer possible.

\section{CONCLUSIONS}

In conclusion, we have demonstrated the versatility of photonic lattices for manipulation of light in conservative and dissipative systems. In particular, we have shown the control of modulational instability and pattern formation by a photonic lattice in a dissipative feedback system. We have identified three important discrete cavity MI control mechanisms: bandgap inhibition of instability modes; seeding of instability patterns by the lattice periodicity; and lattice-induced pattern reorientation. We believe, that our results open new ways for control of the structure of laser modes by embedded photonic crystals. Furthermore, we explore the possibilities for excitation of discrete cavity solitons in such systems.

\section{ACKNOWLEDGEMENTS}

The authors acknowledge the support of the Australian Research Council through Discovery projects, the FrenchAustralian Science and Technology program, and Conseil Régional de Lorraine.

\section{REFERENCES}

[1] J. T. Mok, et al., Nature Physics 2, 775 (2006).

[2] N. K. Efremidis, et al., Phys. Rev. E 66, 046602 (2002).

[3] D. N. Neshev, et al., J. of Nonlin. Opt. Phys. \& Mat. 15, 1 (2007).

[4] D. Gomila, R. Zambrini, and G.-L. Oppo, Phys. Rev. Lett. 92, 253904 (2004).

[5] U. Peschel, O. Egorov, and F. Lederer, Opt. Lett. 29, 1909 (2004).

[6] C. Denz, Ph. Jander, M. Schwab, Ann. Phys. (Leipzig) 13, 391 (2004).

[7] G. Montemezzani, Phys. Rev. A 62, 053803 (2000). 